\title{
LE FILOSOFE E I MAESTRI. QUEL CHE NON ABBIAMO RACCONTATO ${ }^{1}$
}

\author{
MARISA FORCINA \\ Università del Salento
}

\begin{abstract}
Il saggio mostra un altro modo possibile di intendere il rapporto con i maestri. La tesi è che il rapporto di autorità non è, non deve essere vincolato all'uniformità, a un modello unico da conservare in maniera indifferenziata. Deve essere tale da aprire alla libertà dell'altro e costruire la sua autonomia. Il vero maestro è in grado da far dimenticare gli sforzi e le fatiche che hanno aperto alla libertà dell'altro, che non deve porsi nella condizione di eterno debitore e allievoripetitore. Attraverso la lettura di Péguy, la riflessione comune all'interno della Scuola Estiva della Differenza, la prossimità con Françoise Collin, la relazione tra le filosofe e i maestri non trova il suo inveramento in una incondizionata libertà né in una necessità vincolante e sottocondizione, ma trova la sua vitalità in un punto di realtà che è il lavoro ben fatto.
\end{abstract}

PAROLE CHIAVE: Filosofe, maestro, discepolato, autorità, pensiero della differenza, Péguy, Collin.

\section{Las mujeres filósofas y sus maestros. Lo que no hemos contado}

Este ensayo expone un modo alternativo de entender la relación con los maestros. La tesis es que la relación con la autoridad no está, ni debe ser, vinculada con la uniformidad, con un único modelo que debe ser mantenido indiscriminadamente. La autoridad debe ser tal que esté abierta a la libertad del otro y a construir su propia autonomía. El verdadero maestro es capaz de olvidarse del esfuerzo y el gran trabajo que han conducido a la libertad del alumno, a quien no debe poner en una situación de deuda eterna, destinado a ser un discípulo que haga eco de las ideas del maestro. A través de una lectura de Charles Péguy, las reflexiones compartidas en el seno de la Scuola Estiva della Differenza, y la proximidad con el pensamiento de Françoise Collin, sostengo que la relación entre las filósofas y los maestros no alcanza su plenitud en una libertad incondicional ni en una necesidad dependiente y condicionada, sino que encuentra su vitalidad en un punto de realidad que es el trabajo bien hecho.

PALABRAS ClAVE: Filósofas, maestro, discipulado, autoridad, pensamiento de la diferencia, Péguy, Collin.

\section{Women Philosophers and their Teachers. The Untold Story}

This essay proposes a possible way of understanding the student-teacher relationship. I argue that a relationship of authority is not, and should not be, linked with uniformity, with a single

\footnotetext{
${ }^{1}$ Questo studio è stato realizzato nell'ambito del Proyecto de Investigación Científica y Desarrollo Tecnológico Filósofas del siglo XX: maestros, vínculos y divergencias (FFI201230645).
} 
model that must be maintained indiscriminately. Authority should be such that it is open to the freedom of the other to construct her own autonomy. The true teacher is capable of forgetting the effort and hard work that he or she has put into the freedom of the student, whom they should not place in a position of eternal debt, destined to be a disciple echoing her teacher's ideas. Through an analysis of the thought of Charles Péguy, the shared reflexions of the Scuola estiva della Differenza and their similarities with the ideas of Françoise Collin, I argue that the relationship between women philosophers and their teachers is not best realised through unconditional freedom nor through a binding dependence subject to conditions, rather it finds its vitality in a point of reality that is the job well done.

KEY WORDS: Women philosophers, teacher, discipleship, authority, theory of sexual difference, Péguy, Collin.

Nel 1849 George Sand scriveva all'amica Pauline Viardot: "Ah, quanto vorrei avere quindici anni, un maestro intelligente, e tutta la mia vita solo per me! Mi donerei interamente alla musica, ed è in quella lingua che vorrei esprimere i miei sentimenti e le mie emozioni" (Sand, 1991: 63). Quello stesso anno aveva pubblicato Consuelo, il più politico e il più filosofico tra i suoi romanzi, un testo che aveva fatto seguito alla pubblicazione di tanti altri che le avevano dato grande notorietà. La scrittrice era già al massimo della celebrità, eppure, insieme alla giovinezza che non possedeva più, desiderava ancora un maestro. Un maestro intelligente. Il desiderio di un maestro era connesso alla possibilità di esprimersi meglio, a cominciare dalla sfera affettiva. Tra conscio e inconscio, potremmo dire oggi. Dunque i maestri, come in questo caso, non vengono invocati solo per un accesso ai contenuti del sapere, ma per una guida anche nei sentimenti e nelle emozioni. Il panorama è davvero molto più ampio.

Nel 2005 dedicai una "Scuola Estiva della Differenza"2 al tema complesso della relazione con l'altrola in situazioni asimmetriche. In particolare, l'incontro avrebbe dovuto riguardare una relazione non generica, ma quella con l'autorità e voleva fare il punto sulle modalità con cui si incarna il rapporto con colui o colei che riconosciamo e, a volte, disconosciamo come fonte del sapere e dell'agire. In questo tipo di relazione, qualcosa o, meglio, qualcuno o qualcuna, diventa e viene sentito come fonte di vita materiale e spirituale, generatrice a sua volta di un nuovo sapere e di un nuovo fare, nel senso di azione. Ma, a volte, può anche essere paralizzante. Si trattava del rapporto fecondo, ma anche tagliente, con ille maestre, i padri, le madri; un tema complesso, così come complesso risultò anche il titolo: Tra invidia e gratitudine: la cura e il conflitto (cfr. Forcina, 2006).

La sequenza dei termini indicava il percorso: quali sono le nostre relazioni con il sapere e, quindi, come ci si pone in relazione con chi viene percepito come

\footnotetext{
${ }^{2}$ La "Scuola estiva della differenza" è un luogo di formazione nato dalla collaborazione tra l'Università del Salento, l'Università di Roma Tre e il monastero delle Benedettine di Lecce. Dal 2003 si svolge annualmente a Lecce sotto la mia direzione.
} 
maestrola? Come ci si pone nel riconoscimento dei rapporti asimmetrici, in questo difficile incrocio di libertà e necessità?

Perché, se è vero che i maestri ci aprono strade e percorsi di saperi, e quindi di libertà, c'è sempre un rischio con loro: è il rischio della paralisi. Vale a dire che il confronto e la prossimità con l'altrola, che sono maestri nel sapere, ci espone al rischio della cattura o a quello di una ribellione che comporta conflitto o una estrema distanza.

Proponevo, in quella scuola, un ritorno umile a guardare l'invidia e la gratitudine che nascono inevitabilmente in questa relazione, con uno sguardo di esperienza che scendesse all'humus, e quindi si presentasse come ben aderente alla realtà e anche si ponesse "terra terra". Volevo cioè che tale riflessione fosse condotta a partire da concrete esperienze di donne, contro la falsa idea di libertà generata da una ragione supponente o da poteri sacralizzati o dal proliferare delle discipline sempre più "disciplinate" e specializzate che esibiscono il proprio magistero e insegnano un'acribia sempre più lontana dalla realtà.

Tra invidia e gratitudine: la cura e il conflitto programmaticamente prescindeva dalla parità: questa ulteriore deriva che impone alle donne di avere gli stessi standard degli uomini, le stesse percentuali nelle varie modalità di rappresentazione di sé, dall'esercizio della violenza all'esercizio del denaro, dall'esercizio della scienza a quello della cittadinanza, sino alla questione della rappresentanza politica.

Ancora sono convinta che, per avere una società "umana", sia necessario rivisitare le esperienze, i sentimenti, le passioni private e politiche di uomini e donne e vedere come orientare il cambiamento sulla base di consapevolezze espresse e riconosciute. E sulla base degli insegnamenti che non si dimenticano, perché sono venuti da alcuni maestri.

Chi sono i maestrile? Quale genealogia? Solo quella femminile o anche maschile nell'ambito del sapere? E in filosofia qual è il rapporto con i maestri? E soprattutto come si è manifestato?

\section{Una piccola parentesi}

Ricordo uno dei testi di Charles Péguy che più hanno avuto in me risonanza: si tratta de La Città armoniosa, manifesto della differenza. Comincia così: "La città armoniosa ha per cittadini tutti i viventi che sono anime, tutti i viventi animati, perché [...] non conviene che vi siano in essa anime straniere, perché non conviene che vi siano in essa viventi animati stranieri. Così tutti gli uomini di tutte le famiglie, tutti gli uomini di tutte le terre, delle terre a noi lontane e delle terre a noi vicine, tutti gli uomini di tutti i mestieri, dei mestieri manuali e dei mestieri intellettuali, tutti gli uomini di tutti i casali, di tutti i villaggi, di tutti i borghi, di tutte le città, tutti gli uomini di tutti i paesi, dei paesi poveri e dei paesi ricchi, dei paesi deserti e dei paesi popolati, tutti gli uomini di tutte le razze [...] tutti gli uomini di tutte le lingue, tutti gli uomini di tutti i sentimenti, tutti gli 
uomini di tutte le culture, tutti gli uomini di tutte le vite interiori, tutti gli uomini di tutte le credenze, di tutte le religioni, di tutte le filosofie, di tutte le vite, di tutti gli Stati, di tutte le nazioni [...] tutti gli animali sono divenuti cittadini della città armoniosa perché non conviene che in essa vi siano animali stranieri”. Era la città armoniosa dove "tutte le donne, tutti bambini, tutti i malati, tutti i vecchi, tutti gli uomini adulti, validi e giovani, tutti gli animali hanno la loro vita corporea assicurata nella città", dove "le anime individuali diventano ciò che esse sono, ed esse sono indefinitamente varie per i sentimenti della gioia e del dolore e per i sentimenti che non sono né del dolore, né della gioia e tutti questi sentimenti sono tanto sfumati da soddisfare la vita dei sentimenti delle anime individuali" (Péguy, 1984: 47- 48 e 67. Corsivo mio). La litania delle differenze elencate da Péguy indicava come si sarebbe costituita la città. Una città nuova che non avrebbe brandito la bandiera dell'uguaglianza e nemmeno quella della libertà o quella della fraternità, perché aveva avuto modo di constatare che tutte queste bandiere servono ad accentuare piuttosto che far convivere le differenze. Le accentuano al punto che creano guerre in nome della libertà, creano morti e lager in nome dell'uguaglianza e creano separazioni irriducibili in nome della fraternità. Péguy, girando pagina rispetto a tutte queste guerre, proponeva un percorso nuovo di cittadinanza, di civiltà nuova. Proponeva appunto, non una città della legalità, della giustizia e dei diritti, ma una città dove tutti i cittadini diventano ciò che essi sono, e lo diventano se sanno coltivare le loro più grandi differenze.

Nelle parole di Péguy che cadenzano le differenze nel rosario della ripetizione salvifica, non è alcuna lusinga o dover essere, ma l'infinitamente varia possibilità di essere ciascuno se stesso. Nella città armoniosa, infatti, i vecchi non devono diventare giovani né i bambini diventare adulti, né gli animali diventare umani o viceversa, con diritti uguali o equivalenti, ma semplicemente in quella città ciascuno diventa se stesso. E questo non perché autonomia e libertà siano garantiti con norme e legislazioni, ma perché ciascuno sa e può coltivare i propri sentimenti. La città armoniosa è tale perché emozioni e sentimenti sono messi al lavoro e questo lavoro costruisce armonia.

Poi Péguy aggiungeva, con apparente contraddizione, che quella Città armoniosa "non aveva conservato la memoria dei viventi che l'avevano preparata, dei viventi animali a loro vicini o a loro contemporanei, né la memoria delle carità passate, delle giustizie passate, dei tentativi di riparazione degli odi e delle gelosie [...]. Quella città che è armoniosa perché ha dimenticato quelli che l'hanno preparata. E noi che viviamo nella società non ancora armoniosa dobbiamo preparare la città armoniosa tale che ci ignori, che non conosca i nostri sentimenti, che non sappia $i$ nostri sforzi" (71, corsivo mio). Ci sorprende che questa città così bella possa sembrare una città smemorata e quasi irrispettosa e ci sembra un controsenso che proprio chi sa coltivare i propri sentimenti sia poi così distratto rispetto alla propria origine, al punto, sembrerebbe, da 
disconoscere nomi e azioni di chi gli ha preparato ogni agio e libertà. Ma ciò che Péguy non voleva che fossero conservati erano i sistemi e le uniformità costruiti sulle memorie, sulle giustizie, sulle carità e sugli amori che diventano modelli da conservare, sistemi da riprodurre nel medesimo modo e da imporre agli altri. Meglio, allora, l'oblio, meglio dimenticare completamente gli sforzi e la fatica di chi ha preparato quei sistemi, meglio dimenticare liberatori e maestri di libertà piuttosto che essere completamente risucchiati all'interno dell'ingranaggio precostituito. Ma, ed è qui la grande intuizione di Péguy che merita di essere sottolineata con grande rigore, l'azione del dimenticare non è agita dal soggetto che ha la facoltà o meno di dimenticare o ricordare, ma da chi si impegna perché la società armoniosa possa diventare realtà. Il compito supremo dei maestri, il compito supremo di chi desidera e s'impegna perché una società libera possa diventare realtà e continui a restare libera, consiste in questo sforzo: preparare il mondo armonioso di domani, la città libera che sarà abitata dai cittadini liberi di domani, ma agendo in modo tale che questi ultimi non siano costretti in ogni occasione a ricordare quanta fatica è costata quella costruzione. Gli sforzi non dovranno essere contabilizzati, non dovranno essere pesati per presentare il conto agli uomini di domani; i veri maestri non faranno mai pesare i propri sforzi, i propri sentimenti e il proprio nome su chi rappresenta il proprio stesso futuro. Perché il futuro ha da essere libero dai nostri paradigmi e, per avere tale libertà, è meglio che non sappia i nostri sforzi, e dimentichi.

Ecco perché nella filosofia politica dell'autore francese le città diventano armoniose quando finalmente sono libere da chi le ha liberate.

Il testo, dopo aver ri-preso con varie tonalità il senso delle filosofie che, come diceva Péguy, nella loro inascoltata pluralità, nutrono la materia e il movimento, finiva con la costatazione che "i filosofi che riproducono le filosofie che sono già perfette nella loro anima, ossia nella loro carne", sono veramente grandi quando non si affidano agli schemi ideologici o sociologici, ma alla realtà. E concludeva: "I filosofi imparano dai filosofi più vecchi di loro come possono riprodurre le filosofie che hanno perfette nell'anima". Subito dopo, l'autore si spingeva a dire, nelle ultime parole di chiusura, "I filosofi non hanno allievi" (103).

È evidente che si trattava dell'esplicita condanna di ogni ideologia e di ogni possibile radicamento del potere, la negazione di qualunque forma di catechizzazione e di egemonia. Ma era anche l'invito a che "ognuno sia egli stesso ciò che deve essere e che sia realizzato nella sua differenza, nella sua determinazione, nella sua pienezza, nella sua forma più bella" (103. Il corsivo è mio), senza servitù e anche senza dipendenze da maestri catechizzanti.

Il maestro doveva solo essere il primo ad avviare un'azione nella realtà ma non doveva restare l'unico; non doveva pensarsi il migliore e imporre dipendenze. Ecco perché i maestri di Péguy non hanno allievi. Perché non pensano a farsi continuare a farsi ripetere e chiosare dai propri successori. I maestri non impongono subalternità, legami vincolanti e ricordi costanti e 
perenni con dipendenze altrettanto permanenti, ma "sanno restare sulla soglia della vita, sulla soglia di un lavoro radicale che rimette tutto in causa" (Péguy, 1987: 711). Questo perché i maestri devono insegnare non a sostituire la realtà con la propria immagine e il proprio sistema di verità e del vero, devono semmai insegnare a rimettere tutto in questione, non a continuare il sistema, non a capitalizzare persino le rivoluzioni intellettuali, ma a rispettare la realtà, devono insegnare a rispettare ciò che si è e ciò che si deve diventare, restando fedeli alla realtà che è sempre molto più ampia e ricca di ogni sistema (Péguy, 1988: 239240). Per questo nella lezione di Péguy i maestri, che sono coloro che vogliono costruire la città armoniosa, insegnano prima di tutto la libertà a cominciare da quella libertà che si incarna nella libertà di non dover continuamente ricordare le parole o le fatiche o le scelte dei maestri, e insegnano soprattutto a pronunciare parole nuove e ad aprire nuove strade, per questo sembrano non avere, anzi radicalmente non hanno allievi.

\section{Nella filosofia del Novecento}

$\mathrm{Ma}$, a parte Péguy, tutto ciò che è stato fatto, sino al Novecento, nella cultura ufficiale, è stato un lungo e grande tentativo di misurare il mondo e il vissuto maschile e femminile, all'interno delle gerarchie delle generazioni e delle filiazioni e delle citazioni. All'interno dell'ordine simbolico vigente i maestri sono stati sempre riconosciuti come tali e agli allievi e, soprattutto, alle allieve era tutt'al più riservato il compito di mettere in ordine l'opera del maestro. Essere allievi e allieve di un maestro ha significato per molto tempo stargli vicino, sentirsi legati a lui in senso letterale, portarne e proseguirne il segno, che però doveva comunque essere riconoscibile per poter restare anche simbolicamente sempre in mano al potere del maestro. Ne restava escluso, nel senso che era fuori campo, e quindi fuori gioco, non valido, l'ordine simbolico della madre, che Luisa Muraro (1991) ci ha, invece, insegnato a riconoscere e che potrebbe consentire un'organizzazione differente del mondo. L'ordine simbolico della madre ci attesta che la relazione con la madre e, quindi, la relazione con l'altra donna, è la relazione attraverso la quale si apprende a stare al mondo, non è una relazione di potere, ma di autorità. La lingua materna nella quale impariamo a imparare, diventa per questo stesso motivo lingua della conoscenza e garanzia di verità per noi. Lo è non per qualcosa di assoluto e costituivo che le appartiene, ma perché fa parte integralmente di una relazione, quella con la madre, fonte prima di sostegno, nutrimento e insegnamento, fonte di verità per le piccole creature: Mater et magistra? Un interrogativo che evoca quell'enciclica di Giovanni XXIII dove era proposto il valore della Chiesa come relazione tra persone credenti e dove la relazione stessa espressa simbolicamente dalla Chiesa diventando fonte di verità e affermava anche contenuti tradizionalmente più politici, come il valore della libertà economica intesa come libertà dal bisogno e il valore della socializzazione. 
In Muraro, invece, il valore della madre-maestra è nella soggettività incarnata delle donne e si realizza nella relazione con l'altra donna e nella libertà che deriva da questo rapporto di fiducia che autorizza la concretezza di un rendersi conto e di un agire che, muovendo dalla fiducia reciproca, consente il sicuro accesso alla realtà e alla consapevolezza di sé. L'ordine simbolico della madre ha sempre richiamato in me il ritmo di quelle riprese di Péguy che indicavano che i maestri non hanno allievi, perché semplicemente insegnano a stare nella realtà, a parlare, e non a ripetere e far ripetere le proprie parole e i propri nomi.

Muraro ha avuto però anche il merito, agli inizi degli anni '90, di aver messo a tema filosofico-politico questo sapere che si nutre di un ordine simbolico materno e che nella relazione tra donne efficacemente si mostra. Nella tradizione occidentale è apparso qua e là, per esempio nella mistica e nella filosofia di alcuni che vi hanno fatto riferimento -vedi Meister Eckhart e qualcun altro- facendo come un buco nella rete ben tessuta dell'ordine intellettuale e politico vigente e tradizionale. Ma non ha mai rappresentato l'ennesimo sapere togato, strutturante esclusioni, cattedre e poteri; è stato piuttosto una pratica, ossia una esperienza che non è stata teorizzata e riconosciuta ma che, messa alla prova, ha sempre permesso di agire liberamente.

\section{La Scuola estiva della Differenza}

Un banco di prova delle relazione tra le filosofe e i maestri o le maestre è la Scuola estiva della Differenza dell'Università del Salento. Come in ogni scuola, anche in questa è necessario, è indispensabile, è essenziale, è doveroso, è basilare che ci siano dei maestri, e anche delle maestre. In questa Scuola estiva noi privilegiamo l'esperienza femminile che si fa riflessione e parola, ma programmaticamente non escludiamo il rimando e la citazione di riflessioni e di teorizzazioni maschili.

Non è dunque il separatismo postsessantottesco che ci costituisce, ma non è nemmeno una generica volontà di sintesi e di conciliazione. Quello che vogliamo è far emergere una pratica di libertà rispetto a ogni programma totalizzante e, se non è la città armoniosa di Péguy, vuole essere certamente una scuola armoniosa che vuole dare, anche visibilmente, corpo di donna alla riflessione intellettuale delle donne e vuole dare corpo al futuro (Cfr. Forcina, 2013). Il sentire comune, nella scuola, si sedimenta sul rapporto con le maestre e anche con i maestri, ma soprattutto su un regime affettivo capace di accogliere passioni ed emozioni negative e positive, nella consapevolezza che dove ogni investimento affettivo è cancellato, resta solo il disagio e diventa più difficile il percorso di libertà.

Nella Scuola estiva della differenza di Lecce c'è una sorta di mediazione tra tutto ciò che è sapere delle donne e tutto ciò che è cultura tradizionale, politica e scientifica, nella quale anche il sapere delle donne si situa sia pure con scarti per contrapposizioni o per dissensi. Situare nell'alveo della generale tradizione 
culturale le riflessioni femminili non significa necessariamente considerare queste come un'aggiunta o un prolungamento o un inveramento di quella tradizione.

Nella Scuola il sapere femminile prende corpo e si mostra, si relaziona in nuove modalità che riescono a diventare a loro volta percorsi di orientamento e di guida. E' un sapere che si incarna in un modo di essere, in un'autorità femminile che sa indirizzare e permettere una nuova narrazione che diventa conoscenza. Un percorso che educa a una simbolizzazione del mondo che sa rivelare legami con testi e maestri senza esserne mai schiacciata. E' un percorso che insegna come stare in una realtà condivisa, come condividere un pensiero filosofico o politico, come riconoscere l'autorità, ma senza essere ridotti a replicanti accecati dal potere del sapere altrui. E così l'esperienza si fa sapienza e si fa, a sua volta, maestra senza essere mai disincarnata.

Si tratta di un confronto che rischia sui sentimenti e tende a rimuovere ogni fatua beatitudine di tranquillità che deriva dalle verità acquisite e dal potere dei maestri e delle citazioni dotte ed efficaci. Perché se nella cultura ufficiale i rapporti con i maestri sono sedati, accettati e resi indiscutibili, perché tranquillizzano amplificando il sentimento di appartenenza, nella cultura della differenza non sono accettati con il medesimo spirito di squadra; e questo non perché non ne venga riconosciuta l'autorità, ma perché l'ordine simbolico della madre muove dall'esperienza e ad essa chiede conto, genera, come abbiamo visto, contemporaneamente autorità e libertà e, quindi, possibilità di percorsi e discorsi nuovi. Ben sapendo che le appartenenze rischiano di provocare un sentimento di possesso, dipendenza e risentimento, l'autorità che ne prende il posto è frutto di riconoscimento reciproco e promuove la soggettività e non la dipendenza.

Muovendo da queste consapevolezze, già nel titolo della scuola del 2005 era presente la domanda su come il rapporto con l'autorità si debba misurare con l'immediatezza dei sentimenti comuni fatti di invidia e gratitudine. In questo modo si proponeva di imparare a superare gli assoluti della cura e del conflitto che generano paralisi nelle relazioni, a cominciare da quella con il sapere. Ancora una volta le parole rimandavano a corrispondenze, emozioni e saperi che hanno esiti politici concreti. La posta in gioco era ed è sempre la libertà. In gioco è il rapporto con la leadership o con i maestri in senso lato. Infatti, generalmente, sia l'invidia che la gratitudine pongono in una situazione subalterna. Ciò di cui fa esperienza chi invidia non sono tanto le cose o le qualità possedute dall'altrola, cose che chi invidia non ha e di cui non può fare esperienza, ma è il fatto che sta sperimentando e vivendo una privazione. Sa che di fronte alla propria libertà, che appare limitata, c'è un'altra che fruisce di maggiore libertà. La stessa cosa accade per la gratitudine, che costringe al sentimento del rendere grazie, ossia al vincolo di una restituzione del dono ricevuto, sotto altra forma. Ancora una volta la relazione, nell'agire grato, sembra essere privata della libertà, perché si pensa che soprattutto sia importante essere liberi di essere liberi anche dalla gratitudine. 
Il modo più semplice per riacquistare la libertà di cui ci sentiamo privati a causa di questi sentimenti è quello di riaffermarla e ripristinarla. Ma come? La cura dell'altralo ci restituisce alla condizione di soggetti liberi, oppure il conflitto con l'altralo può porci in una condizione di parità. In ambedue i casi la subalternità imposta dalla percezione di un limite alla nostra libertà è superata. Nella cura e nel conflitto generati da un sentimento che si ripresenta reiteratamente e quindi si pone come ri-sentimento, ci si afferma come soggetti liberi.

L'invidia è una forma di ri-sentimento rinnova sentimenti già provati precedentemente e insieme a questi rinnova il dolore e l'avversione contro qualcuno, facendo sì che si sia sempre pronti a rispondere con l'attivazione di un conflitto. Il conflitto però non necessariamente implica la guerra o la fuga, perché essendo espressione di un contrasto tra due autorità diverse, può portare come esito una messa in gioco della libertà attraverso un cambio di ruolo, uno slittamento consapevole dalla propria posizione ad un'altra o attraverso la capacità di una libera mediazione. L'alternativa al risentimento è la gratitudine. Si può provare gratitudine per le cose più elementari: la vita, l'esistenza e il mondo $\mathrm{e}$ per tutti gli altri dati sensibili e sperimentabili intessuti con la carne della realtà. La gratitudine proprio in questi casi porta alla cura del reale e alla cura del mondo, ma quando la gratitudine si esprime verso qualcuno nella forma della cura e viene assolutizzata in quanto tale, rischia di schiacciare il soggetto sotto il suo peso. Ma la gratitudine che porta con sé la cura può anche presentarsi come riconoscenza, ossia come consapevolezza. Nella consapevolezza è implicito un esercizio di sapere, conoscenza e libertà che apre ai soggetti nuove possibilità, se essi sanno stare nella realtà e nell'esperienza. Altrimenti li schiaccia sotto il potere di un obbligo vissuto come debito intimo e profondo.

\section{La risposta di Françoise Collin}

Quando nel 2005 esposi questo percorso a Françoise Collin, lei mi interrogò, come faceva sempre: "E io, che pensi che dovrei dire?". Le proposi un titolo che riprendeva quei giochi di parole che lei amava molto: "Limiti della cura, cura dei limiti". Il titolo rievocava volutamente un suo saggio pubblicato sui Cahiers $d u$ Grif, "Ces études qui sont pas tout. Fécondité et limites des études féministes" (Collin, 1992). Il testo della sua relazione alla scuola estiva è stato pubblicato nel volume uscito nel 2006, con il titolo: "Cura e conflitto a proposito di Le Fils dei Fratelli Dardenne" (Collin, 2007).

Nei Cahiers, Collin insisteva sulla necessità per le donne di essere capaci di imporre limiti alla relazione con l'altro, perché questa non si trasformi in possesso. Anche per la Scuola estiva, mi disse che avrebbe distinto tra care, sorgen e souci, al fine di mostrare come questi termini designano un'alternativa alla forma contrattuale della relazione con l'altro, ma anche presentano dei rischi. 
Infatti se il contratto fissa i limiti dello scambio, la sollecitudine eccede questi limiti e anzi può essere senza limiti, perché non calcola.

Nel chiedersi quale fosse la forma più feconda della relazione con l'altro, il dono incondizionato o il contratto, esplicito o tacito, che fissa i termini dello scambio, Collin quasi esplicitamente sembrava riprodurre la classica contrapposizione tra passione e ragione o tra amore e giustizia così come quella contrapposizione si presenta nella sua urgenza distributiva. In realtà, con uno di quelli scatti inaspettati che la contraddistinguevano, nella sua lezione spostò la questione in avanti, ponendola in un rapporto non teorico con la realtà. La sua conclusione fu che i legami interpersonali hanno bisogno di un qualcosa di più. Il diritto, secondo Françoise, non è ciò che emancipa, ma è ciò che allevia e controlla l'ingiustizia della passione. Ma è anche vero che la passione corregge la rigidità del diritto. Il legame umano si garantisce nella dialettica delicata del diritto e della sollecitudine, del limite e dell'illimitato (Collin, 2007: 55-56). Il diritto, d'altra parte, è solo ciò che consente l'accesso allo spazio pubblico o al riconoscimento di un ruolo. Perché, nel momento in cui l'attività di cura si è ruolizzata, nel momento in cui, come i maestri, le infermiere sono diventate le sole modalità correnti per la cura dell'altro, la sollecitudine si è ridotta a professione e le infermiere diventano la sola risorsa per la fine della vita di coloro che vengono abbandonati dalla propria famiglia. Collin suggeriva che la soluzione non doveva essere quella di aggiungere o di sostituire la cura con la giustizia o con le garanzie sociali. L'ambiguità del dono e della cura potevano essere risolte nella forma del dialogo inteso, in senso arendtiano, come spazio in cui si afferma la presenza e l'interpellanza permanente dell'altro.

La cura, diceva, se trascura i diritti, può diventare una forma sottile di possesso, ma contemporaneamente umanizza i limiti della giustizia e del diritto. Tra il limite e l'illimitato, tra la ragione e la passione, tra giustizia e dono e, potremmo aggiungere, tra le filosofe e i maestri non c'è gerarchia, perché l'uno corregge l'altro, l'uno permette l'altro.

\section{Un esempio inatteso: Le fils, dei fratelli Dardenne}

Françoise Collin ci propose in quella sede il film, Le fils, dei fratelli Dardenne, per mostrarci come si può in una relazione professionale -nella relazione con un maestro- realizzare un legame di dipendenza che non sia univocamente emotivo o passionale o lavorativo o esperenziale. Ci fece notare come, per il maestro, la cura non doveva essere resa possibile dal contratto, ma si doveva articolare nel contratto.

Collin diceva che la trasmissione di un saper fare è il solo modo che rende possibile e realizza un legame altrimenti impossibile con l'altro e che la misura dei gesti condivisi "sostiene e assume ciò che non è divisibile". 
Io utilizzerò le sue indicazioni di fondo e le svilupperò come guida per poter analizzare un'altra situazione, per leggere meglio la relazione che si stabilisce tra le filosofe e i loro maestrile.

Anche le relazioni filosofiche come tutte le relazioni professionali sono attraversate da elementi che sfuggono al registro della professionalità. L'intromissione di un'alterità nella vita del maestro può essere riconosciuta come portatrice di un negativo o di una disgrazia, ma anche di un turbamento positivo fatto di riconoscimento che alimenta il legame con l'altro o che, all'opposto, alimenta il narcisismo. L'intromissione di un legame che non prescinde da sentimenti complessi, quali l'ammirazione e l'attrazione dell'uno per l'altrola, non impedisce di accettare l'alterità. Il problema si pone quando questo legame sembra voler rinunciare al marchio di fabbrica, ossia sembra spezzare la paternità e, con essa, la generazione della vita che viene intesa in generale come creatività; ciò introduce agitazione nella vita di entrambi. Si tratta di un legame asimmetrico.

Il legame asimmetrico può essere fatto di amicizia e repulsione, rifiuto e attrazione, amore e odio. Collin, commentando il film, diceva che qualcosa di "inumano", nel senso di indicibile, legava i due esseri. Mostrava come l'ammirazione ingenua dell'alterità non costituisse il legame. Nel film, infatti il padrone - il maestro - aveva cominciato a legarsi al giovane. I suoi occhi, dietro gli occhiali da vista, sembravano controllarlo e possederlo, ma contemporaneamente si rifiutava di fare da padre al giovane. Quando il maestro scopre che l'assassino di suo figlio è proprio il ragazzo apprendista cui sta insegnando il mestiere, ha una reazione immediata di violenza, ma la capacità di mediazione prende il sopravvento e il sentimento, che in questo caso è di rabbia, ma in altri contesti può essere di affetto, si trasforma, con una schivata di ruoli, in ripresa di contatto con il reale.

Potremmo dire quindi che quando la creatività non campeggia in una solitudine assoluta, sembra essere messa in pericolo, rubata, uccisa dalla presenza dell'altro. Ci si sente troncati, privati della paternità, della titolarità dell'opera o dell'azione. Nasce il conflitto. Eppure il legame si può stabilizzare. Come? Nella trasmissione di un sapere e di un saper fare che deve imparare a prescindere dal sentimento. Allo stesso modo il legame con il maestro genera sentimenti di attaccamento e, contemporaneamente, di ostilità. Esistono due esseri assolutamente separati, eppure una filiazione sostitutiva si stabilisce sempre. Bisogna imparare a trasformare quel tipo di filiazione in un legame sotto condizione. Il limite e l'illimitato, l'incondizionatamente libero e il sottocondizione sono possibili, si incontrano e si incrociano nel lavoro: nel lavoro ben fatto. Sono due in-croci di vissuti: il bene per l'altro e dell'altro, per non essere totalizzante, deve incrociare un punto di realtà, non un sentimento di amore. Tale punto di realtà può essere rappresentato solo dal lavoro: non un lavoro qualsiasi o una fatica qualsiasi, ma il lavoro ben fatto che suscita ammirazione, 
quella sì senza condizione, perché si tratta di un lavoro ben fatto. Lì, dove il bene diventa un fatto, reale come un figlio, reale, in carne ed ossa, il lavoro rappresenta il luogo di un incontro concreto e la libertà si manifesta senza essere risucchiata nel sentimento della gratitudine o dell'odio generato dall'invidia, che vorrebbe erodere completamente persino la presenza dell'altro.

Sembra impossibile che il bene possa entrare nel mondo attraverso il lavoro, che è "una croce", come si dice in gergo, cioè che è pesante come una croce. Eppure è lì la salvezza, una salvezza che redime la fatica e riconsegna l'umano alla sua umanità incarnata fatta di amore, passione, patimento e pena, presenza e assenza. E' lì che il lavoro si trasforma in azione pubblica e riconoscibile.

Hannah Arendt ci ha insegnato che i processi dell'agire possono funzionare solo nello spazio pubblico. E' la politica che rende umano il mondo e sono le azioni del perdonare e del promettere che rendono possibile il futuro. Al contrario, nei processi naturali le azioni dell'uomo sono invece irreversibili e non esiste rimedio alcuno per annullare ciò che è stato fatto (Arendt, 1988: 176). Nel semplice fare, i mezzi della violenza sono gli stessi necessari a ogni fabbricazione, ma anche per disfare ciò che si è fatto non si possono che usare i mezzi della distruzione: "Si disfa ciò che si è fatto come si distrugge un oggetto non riuscito". Se le creazioni artistiche o filosofiche fossero considerate dai propri autori come frutto naturale del loro essere, le azioni conseguenti e le relazioni con gli altri conserverebbero costantemente una dimensione di immediatezza che le renderebbe violente.

Quando la cura o il conflitto sono i mezzi con cui dichiariamo un rapporto di invidia-dipendenza assoluta o di gratitudine assoluta verso i maestri, la violenza occhieggia sempre dietro l'angolo. Resta violenza perché non sa assumere su di sé la libertà di una manifestazione pubblica di quel vissuto che sente invece privatamente come rapporto di dipendenza. La dimensione privata diventa allora totalizzante e si pone come un assoluto.

La filosofia che ha saputo fare i conti con l'assoluto, ha saputo spostare "al limite, la violenza” (cfr. Muraro, 2012). Quando, invece, ha voluto fare dell'assoluto il proprio oggetto o il proprio percorso privato, ne è diventata succube e ha mantenuto se stessa come in un recinto asfittico, anche se molto simile a quello del sacro: "La filosofia -scriveva Françoise Collin in altro contestoè restata vicina al sacro". E poi aggiungeva: "Guardiani del tempio della verità, $o$ della non-verità, ma in ogni caso del rapporto alla verità, lettori e interpreti del Libro laicizzato, garanti dell'integrità del suo corpus i filosofi hanno conservato un qualche rapporto con la casta dei chierici, il loro linguaggio difficile, i loro rituali, i loro privilegi e i loro attributi" (Collin, 1997: 25-26). Il sacerdozio della filosofia non prevede, né spose né figli legittimi. La sua fascinazione non vuole reciprocità, concorrenti o eredi, deve essere assoluta.

La stessa fascinazione si stabilisce tra il maestro che vorrebbe essere senza allievo e restare il solo e come Urano pronto a mangiare i suoi figli per non essere 
spodestato. La filosofa, da parte sua vorrebbe essere senza padre per non essere mangiata da lui.

Nel 2005 a Lecce Françoise Collin ci invitava a leggere il film dei fratelli Dardenne in questa direzione. Coglieva la perturbazione che la figura del maestro sprigionava e ci mostrava come la relazione di dipendenza assoluta avrebbe potuto ricomporsi solo sul registro professionale, sul piano del lavoro che implicava un costante "tenersi a bada". Nel film il padrone aveva, infatti, gesti sottili per tenere a bada il giovane apprendista, non confondeva il saper fare con la paternità, non pagava il panino al ragazzo e non si faceva carico della sua solitudine esistenziale.

Il mondo dei filosofi, come il mondo della falegnameria del film dei Dardenne, era un mondo libero da donne. L'alternativa tradizionale ha affidato alle donne il compito di generare fisicamente il figlio, agli uomini ha lasciato il compito di partorire culturalmente attraverso un legame di filiazione un nuovo soggetto che replica la creatività del maestro. E' possibile realizzare uno spostamento tra natura e cultura?

Proprio attraversando i suggerimenti di lettura di Collin, io credo che si possa rifare un legame senza cancellare ciò che separa e ciò che oppone. Ciò che lega e ciò che slega possono coesistere. Ordini simbolici diversi possono trovare un punto di incontro, possono incrociarsi in un punto. E' difficile ed è penoso, è una croce. E' semplicemente un lavoro, ed è nel lavoro che si ricompone questo difficile equilibrio. Ma è reale, non è utopia.

Per ritrovare un nuovo punto di incontro o una nuova alleanza non è necessaria alcuna dimenticanza o negazione, è necessario solo assumere consapevolezza delle relazioni asimmetriche, simboliche. Ci vuole molta etica in questo difficile rapporto, in questo difficile equilibrio. Ci vuole un'etica dei limiti. Perciò avevo chiesto a Françoise di parlare alla nostra Scuola su: "Limiti della cura, cura dei limiti". Il rapporto con i maestri filosofi non è etico se è incondizionato; se si risolve in una cura incondizionata, fa perdere ogni libertà. $\mathrm{E}$ politico ed è etico, invece, se viene corrisposto nei limiti rigorosi di un contratto, come diceva Françoise Collin, o se è pubblico. Oppure se ha una regola come quella che indica Muraro (2012: 71), che non si riferisce al lavoro regolamentato in maniera professionale, ma alla sapienza antica di donne che in cucina hanno sempre saputo regolare il sale, con quel "quanto basta" sempre differente per cibi differenti.

L'affettività è altro dal far essere che è il lato costitutivo della filosofia e del lavoro. Si può non dare un sentimento appassionato di affetto, ma dare un saper fare e, al contrario, in modo immorale si può dare affetto negando l'insegnamento al saper fare (si vedano a questo proposito le lettere di Heidegger a Arendt, 2007).

Il dono della filosofia non è dono d'amore sia pure intellettuale tra i maestri e le filosofe, ma è un dono sotto condizione, un dono che stabilisce una frontiera; 
ma proprio per questo è più valido. È il dono di cui aveva parlato Péguy nella città armoniosa, il dono di lavoro che lascia intatta la libertà dell'altro. E' dono che si sa regolare con il sale dell'oblio. È il dono limitato che lascia alla singolarità dei propri destini il maestro e la filosofa, il padrone e l'apprendista.

Il film dei Dardenne finisce con una scena dove il giovane apprendista comprende ciò che il maestro sapeva già, e cioè che il proprietario della falegnameria era il padre del ragazzo che lui stesso aveva ucciso. Nel film, davanti a tale verità si scatena la violenza. L'assoluto, come Dio, o come la verità, se non è incarnato in qualcosa o qualcuno è tremendum, come il sacro nella definizione di Rudolf Otto (2011), è violent, come nel testo di Luisa Muraro (2012). La differenza tra i due è che Otto associa il sacro alla violenza e al terrore di fronte all'inquietante, emozione irrazionale costituita dall'inavvicinabile per eccellenza in cui si costituisce la potenza divina, che è essenzialmente forza, energia, vitalità, ma anche veemenza che si manifesta nelle rappresentazioni simboliche dell'ira di Dio e delle sue svolte apocalittiche. Muraro, invece, dando corpo al sacro, che per lei è costituito dalla relazione tra donne, si affida alla misura. Per Muraro, di fronte a una società in cui è venuta meno la narrazione salvifica del contratto sociale e di fronte all'assoluto di una politica impotente rispetto ai potenti e incapace di risolvere i problemi, non c'è che da rispondere con tutta la propria forza di resistenza e di opposizione, ma sapendo incarnare questa forza e affidandosi a una antica sapienza femminile: "Quando è il caso di decidere come comportarci, regoliamoci come fanno le cuoche con il sale: 'Quanto basta'. La formula che ho trovato dice: quanto basta per combattere senza odiare, quanto serve per disfare senza distruggere" (2012: 71-72).

Nel film, alla scena violenta della verità nella sua più assoluta crudeltà segue quella del giorno dopo: nessun gesto può cancellare la ferita. Ma il lavoro riprende.

Un legame come quello tra le filosofe e i maestri, che mette al lavoro la verità, come ogni legame, si costruisce proprio su una ferita forte e precisa. Entrambi hanno perduto irrimediabilmente un mondo, quello della filosofia del sistema e dell'io creatore del mondo o ordinatore, ma entrambi possono ricostruire il legame col mestiere che hanno imparato e insegnato. Possono trovare quel punto d'incontro che segna e permette la differenza e, con tutti i limiti di quella differenza, dare ancora una possibilità all'etica, cioè in generale al rapporto con l'altro.

Ancora una volta, anche se non direttamente, il pensiero filosofico di Collin ci sorprende per la sua continuità e fedeltà: già nei suoi Cahiers aveva scritto: "Ma voler durare vuol anche dire, per ognuno di noi, imparare a morire, abbandonarsi a una filiazione che dà luogo all'altro: nominare ed essere nominate senza la garanzia astratta del lignaggio patriarcale" (Collin, 1986: p. 82. Traduzione mia). 
Morire fa rima con dormire. Chi dorme sembra quasi morto, ma non lo è. Dormire non è solo una citazione di morire: dormire non è scomparire, ma aver cura di sé, dormire non è svanire dal mondo, ma darsi un tempo per riposare, per non invadere con il proprio sé e le proprie azioni il giorno degli altri, e di quelle altre che sono venute dopo. Pochissime filosofe hanno conosciuto il sonno dei filosofi maestri e quell'alternanza di sonno e di veglia che porta a nominare ed essere nominate nella reciprocità di un dialogo che costruisce la città armoniosa fatta di differenze costitutive della cui espressione libera abbiamo sempre avuto desiderio, e che è reale e possibile a patto che il patriarcato impari anche a dormire, in modo semplice e sano, in modo normale, di quella normalità che sa $\mathrm{e}$ fa la differenza.

\section{TESTI CITATI}

Arendt, Hannah (1988), Vita activa. La condizione umana, Sergio Finzi (trad.), Milano, Bompiani.

Arendt, Hannah e Martin Heidegger (2007), Lettere 1925-1975 e altre testimonianze, a cura di Ursula Ludz e Massimo Bonola, Torino, Einaudi.

Collin, Françoise (1986), "Un héritage sans testament”, Les Cahiers du Grif, 34.

-(1992), “Ces études qui sont pas tout. Fécondité et limites des études féministes", Les Cahiers du Grif, 46.

-(1997), "L'oblio di una vecchia questione filosofica", Françoise Collin e Marisa Forcina, La differenza dei sessi nella filosofia, nodi teorici e problemi politici, Lecce, Milella.

Forcina, Marisa (ed.) (2006), Tra invidia e gratitudine: la cura e il conflitto, Lecce, Milella.

-(2013), Diamo corpo al futuro, Lecce, Milella.

Muraro, Luisa (1991), L'ordine simbolico della madre, Roma, Editori Riuniti.

-(2012), Dio è violent, Roma, Edizioni nottetempo.

Otto, Rudolf (2011), Il sacro. Sull'irrazionale nell'idea del divino e il suo rapporto con il razionale, a cura di Aldo Natale Terrin, Brescia, Morcelliana.

Péguy, Charles (1984), La città armoniosa, a cura di Leonardo La Puma, Lecce, Milella.

-(1987) Casse-cou. Euvres en prose complètes, volume I, Parigi, Pléiade.

-(1988), Lo spirito di sistema, a cura di Marisa Forcina, Lecce, Milella.

Sand, George (1991), Correspondance, tomo IX, Parigi, Garnier. 